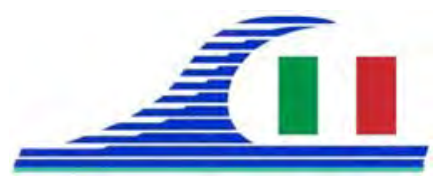

Conférence Méditerranéenne Côtière et Maritime EDITION 3, FERRARA, ITALIA (2015)

Coastal and Maritime Mediterranean Conference

Disponible en ligne - http://www.paralia.fr - Available online

\title{
La méthode de décomposition du domaine : application à un problème de soutirage
}

\author{
Soumia MORDANE ${ }^{1}$, Khalid ADNAOUI $^{2}$, Mohamed LOUKILI ${ }^{1}$, \\ Noureddine TOUNSI ${ }^{2}$, Mohamed CHAGDALI ${ }^{1}$
}

\author{
1. LPPPC, Faculté des Sciences Ben M’Sik, Casablanca -Maroc. \\ mordanesoumia@yahoo.fr \\ 2. MITI, Faculté des Sciences Ben M’Sik, Casablanca. Maroc.
}

\section{Résumé :}

Dans ce travail, on présente une méthode de décomposition de domaine pour résoudre les équations de Navier Stokes. Deux applications sont présentées pour illustrer ses avantages: la première concerne le jet par le fond dans un canal à surface libre et la seconde est celle du soutirage latéral. Ces deux applications concernent la problématique de la création de la circulation artificielle des masses d'eau en vue d’homogénéiser les paramètres physique du milieu.

Dans l'application de la méthode de décomposition, le domaine d'étude est décomposé en sous domaines en vue de mieux prendre en compte des processus physiques et les méthodes numériques adaptés à leurs applications. Deux méthodes numériques sont utilisées, une méthode des différences finies près de la paroi et une méthode particulaire dans les zones de forte circulation. Dans les interfaces des sous domaines, on utilise des conditions de couplage par la méthode Particule- Maillage.

Mots-clés : Navier-Stokes, Particulaires, Différences finies, Soutirage.

\section{Introduction}

La résolution directe par la méthode des différences finies des équations de Navier Stokes dans un domaine, pose un certain nombre de difficultés pour les grands nombres de Reynolds. D’autres méthodes sont mieux adaptées à traiter les écoulements de fluides visqueux dans le cas des fortes recirculations de la vitesse. Parmi ces méthodes, on cite les méthodes particulaires (HUBERSON \& CHOQUIN, 1988). La combinaison de ces deux méthodes permet d'améliorer la résolution des équations de Navier Stokes en particulier pour les grands domaines d'étude. Ce travail utilise la méthode de décomposition du domaine pour deux applications liées au problème de stratification des milieux dans un bassin: Utilisation d'un jet par le fond et le soutirage latéral.

\section{Méthode de décomposition du domaine}

On cherche à résoudre les équations de Navier Stockes du système d'équations suivant: 
Côtes méditerranéennes menacées :

Risques et défis dans le contexte du changement climatique

$$
\begin{aligned}
& \frac{\partial \omega}{\partial t}+(\vec{V} \cdot \vec{\nabla}) \omega=\frac{1}{\mathfrak{R}_{e}} \Delta \omega+\frac{1}{F r^{2}}\left[\vec{\nabla} C \wedge \frac{\vec{g}}{\|\vec{g}\|}\right] \cdot \vec{k} \\
& \Delta \psi=-\omega \\
& \vec{V}=\vec{\nabla} \wedge(-\psi \vec{k}) \\
& \frac{\partial C}{\partial t}+(\vec{V} \cdot \vec{\nabla}) C=\frac{1}{\Re e S c} \Delta C
\end{aligned}
$$

Avec $\vec{\nabla}$ et $\Delta$ sont respectivement l'opérateur gradient et l'opérateur Laplacien, $\vec{k}$ est le vecteur directement perpendiculaire au plan de l'écoulement, $\overrightarrow{\mathrm{U}}$ est le vecteur vitesse, $\psi$ est la fonction de courant, $\omega$ est la fonction tourbillon tel que $\vec{\omega}=\omega \vec{k}=\vec{\nabla} \wedge \vec{U}, C$ est la concentration du polluant et $\vec{g}$ est l'accélération de la pesanteur. $\mathfrak{R} e, S c, F r$ représentent respectivement les nombres de Reynolds, de Schmidt et de Froude.

\subsection{Application au problème d'un diffuseur sur le fond}

Sur le fond d'un canal rectangulaire (Longueur= $6 \mathrm{~m}$, profondeur $\mathrm{l}=4 \mathrm{~m}$ ), on a placé un diffuseur de diamètre $1 \mathrm{~m}$. Le domaine d'étude est limité par deux frontières latérales, une surface libre plane et un fond horizontal imperméable. (voir figure 1). Les équations à résoudre sont les équations 1-4. Les nombres adimensionnels choisis sont $\mathrm{F}_{\mathrm{r}}=18$, $S_{c}=1.5$. Le nombre de Reynolds est un paramètre d'étude. Dans l'application de la méthode de décomposition du domaine, on partitionne $\Omega$ en deux sous domaines (figure 2). Dans le sous domaine $\Omega 1$, on choisit de résoudre les équations 1-4 par une méthode des différences finies. Dans $\Omega 2$, on résout le problème par la méthode particulaire. Ce choix est justifié par l'aspect de l'écoulement. En effet, Dans ce sous domaine, c'est l'aspect de forte recirculation de la vitesse qui domine et la méthode des différences finies est moins adaptés à traiter cet aspect du mouvement. La méthode particulaire est une méthode lagrangienne adapté à traiter les problèmes de convection en régime turbulent (HUBERSON \& CHOQUIN, 1988). Le couplage se fait par une méthode de particule maillage.

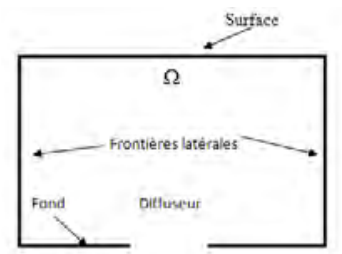

Figure 1. Domaine d'étude $\Omega$.

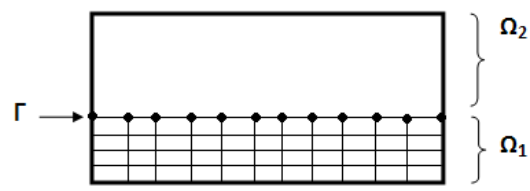

Figure 2. Subdivision du domaine $\Omega$.

On présente respectivement les résultats dans le sous domaine 1 (figure 3a), à l'interface au moment où le fluide commence à quitter le domaine $\Omega 1$, par un couplage faible (figure 3b) et dans le sous domaine 2 (figure 3c) pour un nombre de Reynolds égale à 1000. Les unités des axes sont adimensionnelles rapportées au diamètre du jet. 
On constate que la reprise des informations à partir du sous domaine 1 pour le sous domaine 2 se fait d'une manière régulière et continue. Cette constatation est justifiée dans la figure 4 où le champ de vitesse est représenté dans tout le domaine. L’intensité du champs des vitesses est sans dimension.

\subsection{Application au soutirage sélectif}

Le domaine considéré est rectangulaire, rempli d'eau, de largeur 4m et longueur $6 \mathrm{~m}$. Un jet est imposé en amont et un soutirage dans la partie aval. La configuration géométrique est présentée sur la figure 5. L'objectif est d'analyser l’impact de ce mécanisme sur la recirculation de l'eau en vue d'éliminer les phénomènes d'eutrophisation.

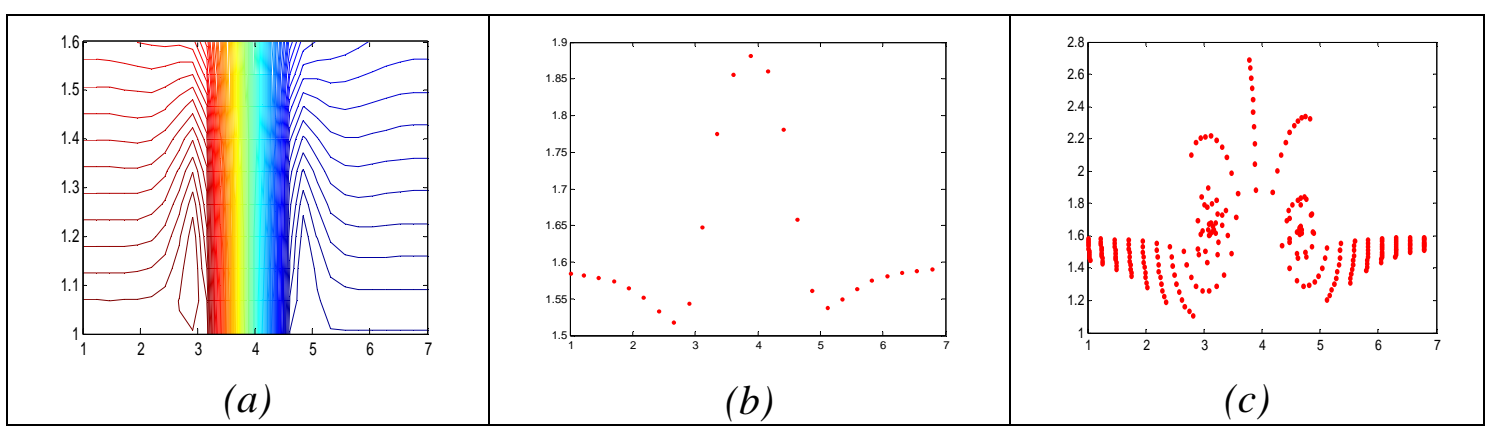

Figure 3. (a) Iso-concentrations dans le sous domaine $\Omega 1$; (b) Position des particules du jet ; (c) Illustration de la méthode de projection.

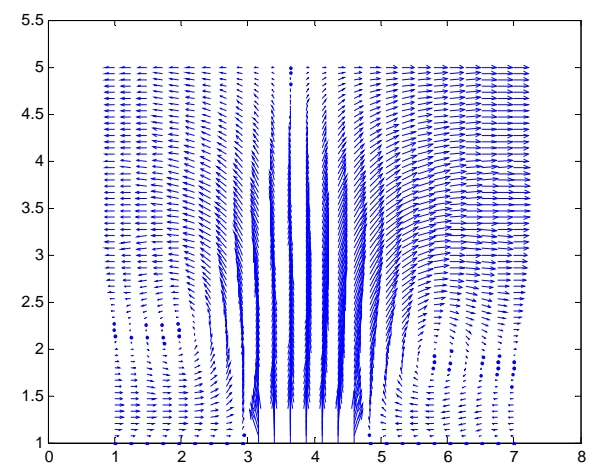

Figure 4. Champ des vitesses dans le domaine d'étude.
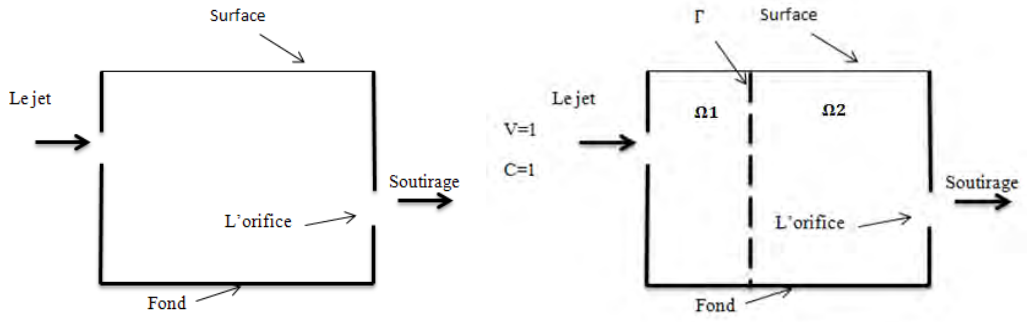

Figure 5. Le domaine d'étude $\Omega$. 
Côtes méditerranéennes menacées :

Risques et défis dans le contexte du changement climatique

On présente sur la figure 6 les lignes de courant pour un nombre de Reynolds $\mathfrak{R}=1000$. La reprise des données calculées par la méthode des différences finies du domaine 1 est régulière vers le domaine 2 où le calcul se fait par la méthode particulaire. Ce raccordement est montré sur la figure 7.

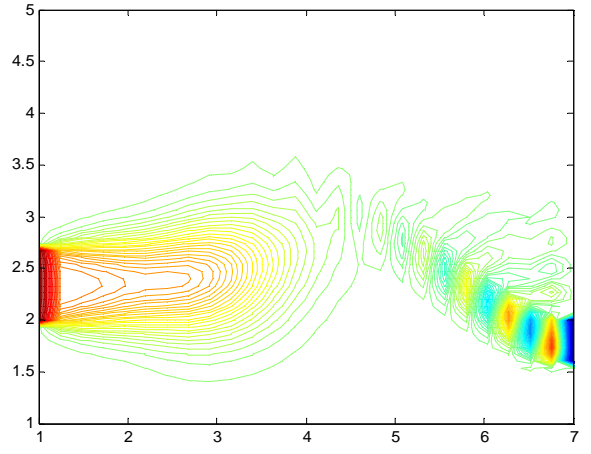

Figure 6. Soutirage a l'instant $t=10 \mathrm{~s}$.

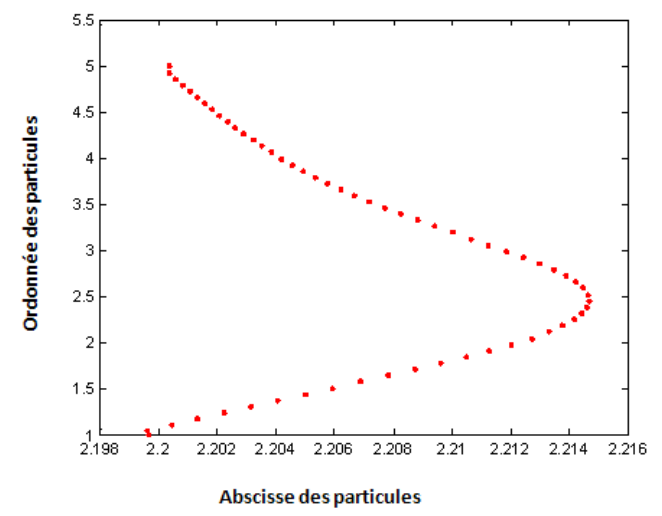

Figure 7. Position des particules du jet.

\section{Conclusion}

La méthode de décomposition du domaine que nous avons proposé permet d'analyser et de simuler des problèmes à grands nombre de Reynolds. Elle est bien adaptée aux problèmes des écoulements confinés et aux écoulements externes en présence des parois. Elle a été appliquée à deux problèmes régis par deux processus physique différents: le premier est régit par la gravité et le second est régit par la flottabilité. L'extension de cette méthode à des problèmes de natures différentes constitue l'une de nos perspectives. Son principal avantage est d'effectuer des résolutions non linéaire dans des grands domaines géométriques.

\section{Références bibliographiques}

ADNAOUI K, TOUNSI N., CHAGDALI M., MORDANE S. (2014). The method of decomposition domain for the digital modelling of a jet. American Journal of Applied Mathematics, Vol. 2(2), pp 64-68. http://dx.doi.org/10.11648/j.ajam.20140202.14

HUBERSON S., CHOQUIN P. (1988). Particles simulations of viscous flow. Computers and Fluids n ${ }^{\circ}$ 2, pp 397-410.

MANGOUB G. (2007). Méthode de sous domaine et de décomposition de champs de vitesse :Application aux écoulements à surface libre. Thèse de Doctorat d'état à l’Université Hassan II Mohammedia , Faculté Ben M’Sik.

ROUX Y. (1999). Etude de l'amortissement visqueux dans les problèmes de tenue à la mer d'un flotteur immergé. Thèse de Doctorat, Université du Havre. 